ESAIM: PROCEEDINGS, April 2009, Vol. 26, p. 150-161

H. Ammari, Editor

\title{
RECENT PROGRESS ON FREQUENCY DIFFERENCE ELECTRICAL IMPEDANCE TOMOGRAPHY
}

\author{
Jin Keun SEO ${ }^{1}$, Bastian Harrach ${ }^{2}$ And Eung Je WoO ${ }^{3}$
}

\begin{abstract}
Although time-difference EIT(tdEIT) has shown promise as a medical EIT imaging technique such as monitoring lung function, static EIT has suffered from forward computational model errors including boundary geometry and electrode positions uncertainty combined with the ill-posed and highly nonlinear nature of the corresponding inverse problem. Since 1980s, there has been great endeavor to create forward computational models with the necessary accuracy required for EIT reconstruction, but these efforts were not successful in clinical environment. This is the main reason why we consider frequency-difference EIT (fdEIT) where we take advantage of frequency dependance of biological tissue by injecting currents with at least two different frequencies. In this article, we review recent progress in fdEIT.
\end{abstract}

\section{INTRODUCTION}

Viewing the human body as a composition of resistance and reactance, we can characterize electrical properties of a living organism by measuring the ratio of an externally applied current to the resulting voltage. The electrical conductivity and permittivity values of biological tissues and organs change with their physiological and pathological conditions and thus provide useful diagnostic information. Hence, the non-invasive measurement of the bioelectric impedance inside the human body has been an important research topic in biomedical engineering.

Electrical Impedance Tomography(EIT) is a non-invasive imaging technique which aims to provide the crosssectional distribution of electrical impedance inside the human body. In EIT, we attach surface electrodes (typically 8 to 256) on the boundary of the subject, inject linearly independent patterns of sinusoidal currents in the frequency range of $50 \mathrm{~Hz}$ to $500 \mathrm{kHz}$, and measure the induced complex voltages. Since the relationship between the applied current and the resulting voltage data provide the electrical propensity of the subject, we use all available distributed current patterns and the measured voltage data set to reconstruct cross-sectional images of the conductivity and/or permittivity distribution inside the subject. This EIT technique has received considerable attention over the past two decades. Several review papers describe numerous aspects of the EIT technique $[5,8,24,29,37]$, and mathematical theory was developed to support EIT system $[1,10,16,23,26,27$, $34-36]$.

In order to make a static EIT image reconstruction algorithm reliable as a medical EIT imaging technique, one needs to construct a forward computational model with the same geometry as the imaging object and the accurate electrode positions. Barber and Brown [15] pointed out that, in order to obtain useful EIT images,

\footnotetext{
1 Department of Computational Science \& Engineering, Yonsei University, Korea

2 Institute for Mathematics, Johannes Gutenberg-Universität, 55099 Mainz, Germany

3 College of Electronics and Information, Kyung Hee University, Korea
} 
electrode positions should be determined within $0.1 \mathrm{~mm}$ accuracy when electrodes are spaced $100 \mathrm{~mm}$ apart. In clinical environments, achieving this accuracy in the forward modeling would be very difficult with a reasonable cost. This requirement of accuracy is related to the fundamental shortcomings of the corresponding inverse problem that has low sensitivity of measured data to a local change of conductivity, relatively high sensitivity of measured data to boundary geometry errors and electrode positions uncertainty, and high nonlinearity between data and internal conductivity distribution. Without dealing with these undesirable influences of modeling errors, the static EIT imaging cannot be successful in clinical environments.

In time difference EIT(tdEIT), we reconstruct the temporal change of the complex conductivity distribution using the time difference between two consecutive measured voltage data sets. tdEIT takes advantage of alleviating modeling errors contained in the voltage data set since the time difference between two consecutive boundary voltage data sets may cancel out boundary geometry errors and electrode positions uncertainty. tdEIT imaging was first proposed by Barber and Brown [4] using the backprojection method, and Cheney et al. [9] used the one-step Newton method for tdEIT. tdEIT has been applied to the monitoring of heart function, blood flow, and emptying of the stomach. Most of the published researches on phantom experiments essentially were based on tdEIT techniques since their results used the measured voltage data set corresponding to a homogeneous background instead of using a computed voltage data set for a forward model [15, 25, 28].

In the case of tumor imaging including breast tumor and in stroke type detection, we cannot use the tdEIT method since a reference measurement of the voltage data is not available, and any method using the computed reference voltage with a forward model can not be reliable due to the modeling errors mentioned before. To deal with this problem, we consider frequency-difference EIT (fdEIT) where we inject currents with at least two different frequencies. In fdEIT, it is essential to use the weighted difference of boundary voltage data to produce an image of frequency-dependent changes of the internal complex conductivity distribution. Compared with tdEIT, fdEIT does not require a reference data set from the past.

This lecture note focuses on robust reconstructions (instead of high resolution) under practical environments having various technical limitations due to the data collection equipment and the fundamental limitations of its inherent nature. We describe the mathematical formulation of single and multi-frequency EIT in clinical environments, image reconstruction algorithms, measurement techniques, and show examples of EIT images.

\section{INVERSE PROBLEMS OF FDEIT}

\section{1. fdEIT model}

The human body can be viewed as a mixture of resistors and capacitors. We begin with reviewing a circuit model containing resistors, capacitors, and a cosinusoidally time-varying current source. If the current source in the circuit is given by $I_{\omega}(t)=I \cos (\omega t)$ where $I$ is the amplitude and $\omega$ is the angular frequency, then the resulting voltage $V_{\omega}(t)$ between two points in the circuit is also time-harmonic with the same angular frequency $\omega$. The relation between $I_{\omega}(t)$ and $V_{\omega}(t)$ is given by

$$
R \dot{I}_{\omega}(t)+\frac{1}{C} I_{\omega}=\dot{V}_{\omega}(t)
$$

where $R$ is the total resistance of the circuit and $C$ is the total capacitance. $R$ and $C$ depend on the circuit topology, the position of the current source and the two points between which the voltage is measured. The resulting voltage can be written as

$$
V_{\omega}(t)=R I \cos (\omega t)+\frac{I}{C \omega} \sin (\omega t)=\tilde{V}_{\omega} \cos \left(\omega t-\phi_{\omega}\right),
$$


where $\tilde{V}_{\omega}=\sqrt{(R I)^{2}+\frac{I^{2}}{C^{2} \omega^{2}}}$ is the amplitude and the phase shift $\phi_{\omega}$ satisfies $\tan \phi_{\omega}=\frac{1}{R C \omega}$ and $0 \leq \phi_{\omega} \leq \frac{\pi}{2}$. Introducing the complex potential difference $u_{\omega}=\tilde{V}_{\omega} e^{-i \phi}$, we have

$$
\left[R+\frac{1}{i \omega C}\right] I e^{i \omega t}=u_{\omega} e^{i \omega t}, \quad \text { or } \quad R+\frac{1}{i \omega C}=\frac{u_{\omega}}{I} .
$$

Hence, we can determine the complex total impedance $Z:=R+\frac{1}{i \omega C}$ from the relationship between $I$ and $u_{\omega}$.

Next, we consider a two or three dimensional model inverse problem in EIT. Let the subject occupy a two or three dimensional region $\Omega$ bounded by its surface $\partial \Omega$. Assume that the boundary $\partial \Omega$ is connected and smooth. In EIT, we attach copper electrodes $\mathcal{E}_{1}, \ldots, \mathcal{E}_{L}$ on $\partial \Omega$. With these $L$ surface electrodes, we usually apply $L$ different time-harmonic electrical currents using the $L$ pairs of electrodes $\left(\mathcal{E}_{j}, \mathcal{E}_{j+1}\right), j=1, \ldots, L$ to inject the sinusoidal current $I \cos (\omega t)$. Here, we denote $\mathcal{E}_{L+1}=\mathcal{E}_{1}$. Although the injection current using the pair $\left(\mathcal{E}_{L}, \mathcal{E}_{1}\right)$ is a linear combination of the other injection currents using pairs $\left(\mathcal{E}_{j}, \mathcal{E}_{j+1}\right), j=1, \ldots, L-1$ mathematically, we always inject this redundant current for compensation of systematic errors in practice.

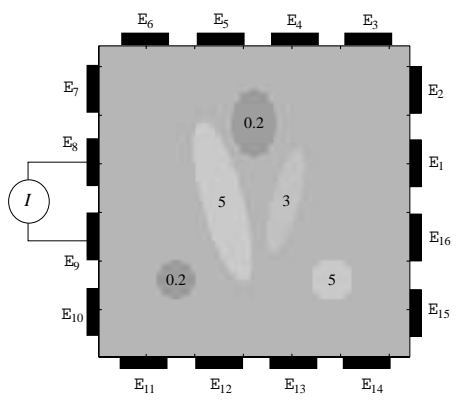

(a)

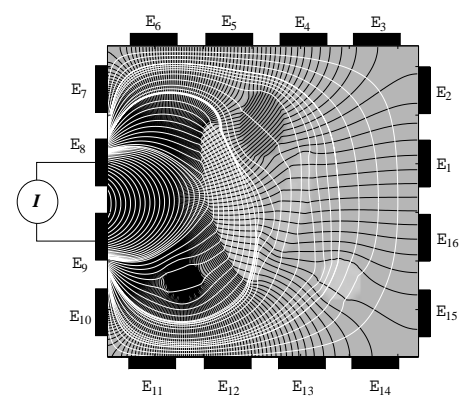

(b)

FiguRE 1. (a) Surface electrodes $\mathcal{E}_{j}, j=1, \ldots, 16$, are attached on the boundary of a simplified rectangular model with a given conductivity distribution. (b) We inject the AC current $I \cos (\omega t)$ using the electrodes $\mathcal{E}_{8}$ and $\mathcal{E}_{9}$.

Let $V_{\omega}^{j}(\mathbf{r}, t)$, be the corresponding electric potential to the current $I \cos (\omega t)$ injected through the pair of electrodes $\left(\mathcal{E}_{j}, \mathcal{E}_{j+1}\right)$. Writing $V_{\omega}^{j}(\mathbf{r}, t)=\Re\left\{u_{\omega}^{j}(\mathbf{r}) e^{i \omega t}\right\}$ and assuming that $\frac{\omega}{\pi} \leq 500 \mathrm{kHz}$ and the diameter of the subject is less than $2 \mathrm{~m}$, the time-harmonic voltage $u_{\omega}^{j}$ satisfies

$$
\left\{\begin{array}{rlrl}
\nabla \cdot\left(\gamma_{\omega} \nabla u_{\omega}^{j}\right) & =0 & & \text { in } \Omega, \\
\left.\left(u_{\omega}^{j}+z_{k} \gamma_{\omega} \frac{\partial u_{\omega}^{j}}{\partial \mathbf{n}}\right)\right|_{\mathcal{E}_{k}} & =U_{\omega}^{j, k} & & \text { for } k=1, \ldots, L, \\
\gamma_{\omega} \frac{\partial u_{\omega}^{j}}{\partial \mathbf{n}} & =0 & & \text { on } \partial \Omega \backslash \cup_{k=1}^{L} \mathcal{E}_{k}, \\
\int_{\mathcal{E}_{k}} \gamma_{\omega} \frac{\partial u_{\omega}^{j}}{\partial \mathbf{n}} d s & =0 & & \text { if } k \in\{1, \ldots, L\} \backslash\{j, j+1\}, \\
\int_{\mathcal{E}_{j}} \gamma_{\omega} \frac{\partial u_{\omega}^{j}}{\partial \mathbf{n}} d s & =I=-\int_{\mathcal{E}_{j+1}} \gamma_{\omega} \frac{\partial u_{\omega}^{j}}{\partial \mathbf{n}} d s .
\end{array}\right.
$$

where $z_{k}$ is the contact impedance of the $k$-th electrode $\mathcal{E}_{k}, U_{\omega}^{j, k} \in \mathbb{C}, \mathbf{n}$ is the outward unit normal vector on $\partial \Omega$ and $\gamma_{\omega}=\sigma(\mathbf{r}, \omega)+i \omega \epsilon(\mathbf{r}, \omega)$ is the complex conductivity which depends on the position $\mathbf{r}=(x, y, z)$ and the angular frequency $\omega$. Setting a reference voltage by assuming that $\int_{\partial \Omega} u_{\omega}^{j} d s=0$, we can obtain a unique solution $u_{\omega}^{j}$ of (1) [33]. Throughout this work, we neglect the contact impedance and assume that $\left.u_{\omega}^{j}\right|_{\mathcal{E}_{k}}=U_{\omega}^{j, k}$ for simplicity. 


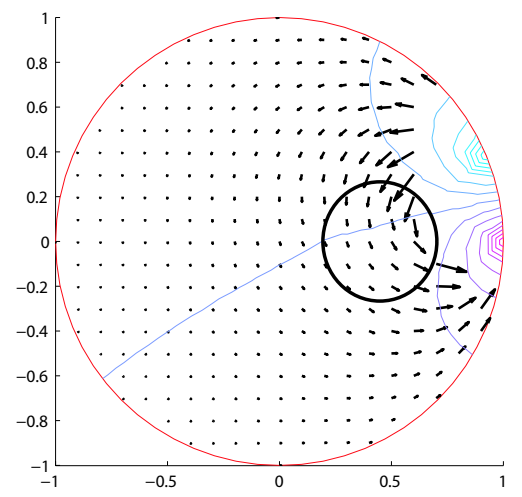

(a)

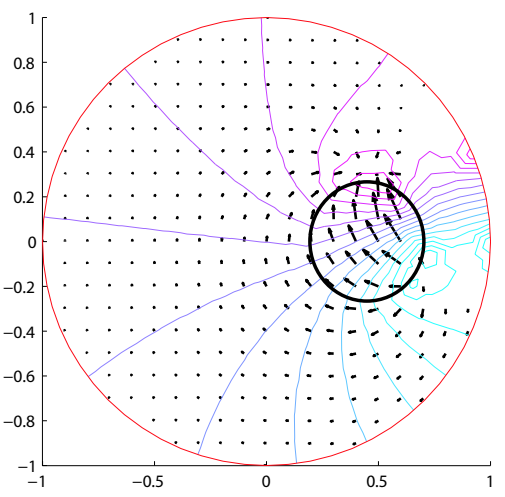

(b)

FIGURE 2. Equipotential lines (solid lines) and electric field streamlines (arrows) of the complex potential $u_{\omega}$ in $(1)$ at $\omega / 2 \pi=100 \mathrm{kHz}$ subject to an injection current between an adjacent pair of electrodes. Complex conductivity values of the anomaly and the background were related to those of banana and saline, respectively. (a) Real part $\left(\Re\left\{u_{\omega}\right\}\right)$ and (b) imaginary part $\left(\Im\left\{u_{\omega}\right\}\right)$. This figure is quoted from [31].

In fdEIT, we measure the following data set $\mathbb{U}_{\omega}$ for various angular frequencies:

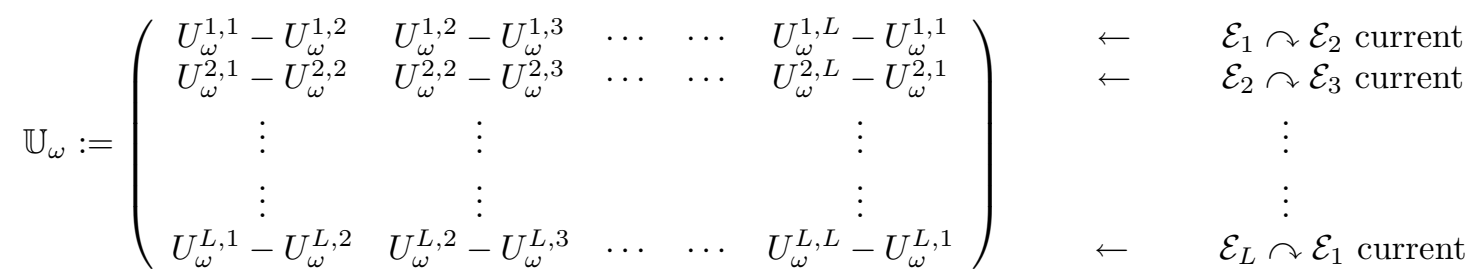

We try to visualize the change of $\gamma_{\omega}=\sigma_{\omega}+i \omega \epsilon_{\omega}$ due to the change of the angular frequency $\omega$.

If the complex conductivity at two frequencies, $\gamma_{\omega_{1}}$, resp., $\gamma_{\omega_{2}}$, is spatially constant in the subject $\Omega$, then $\gamma_{\omega_{1}} u_{\omega_{1}}^{j}=\gamma_{\omega_{2}} u_{\omega_{2}}^{j}$ and

$$
\frac{\gamma_{\omega_{2}}}{\gamma_{\omega_{1}}}=\frac{\int_{\Omega} \gamma_{\omega_{1}} \nabla u_{\omega_{1}}^{j} \cdot \nabla u_{\omega_{1}}^{k} d \mathbf{r}^{\prime}}{\int_{\Omega} \gamma_{\omega_{2}} \nabla u_{\omega_{2}}^{j} \cdot \nabla u_{\omega_{2}}^{k} d \mathbf{r}^{\prime}}=\frac{U_{\omega_{1}}^{j, k}-U_{\omega_{1}}^{j, k+1}}{U_{\omega_{2}}^{j, k}-U_{\omega_{2}}^{j, k+1}}
$$

This means that we can obtain the quotient $\frac{\gamma_{\omega_{2}}}{\gamma_{\omega_{1}}}$ from the data $\mathbb{U}_{\omega_{1}}$ and $\mathbb{U}_{\omega_{2}}$ without any knowledge of the geometry of $\Omega$ or of the electrode positions when the subject is homogeneous.

\subsection{Feasibility of fdEIT}

For simplicity, we assume that the subject occupies a bounded smooth domain $\Omega$ and that it comprises a conductivity anomaly $D$ such that, for each frequency $\omega$, the complex conductivity distribution $\gamma_{\omega}(\mathbf{r})=$ $\gamma(\mathbf{r}, \omega)=\sigma(\mathbf{r}, \omega)+i \omega \epsilon(\mathbf{r}, \omega)$ is constant in the background $\Omega \backslash \bar{D}$, and constant in the anomaly $D$. The respective constants may change with $\omega$.

Assume that $u_{\omega}^{j}$ is the solutions of (1). For $\mathbf{r} \in \partial \Omega$ and a sufficiently small $s>0$, the complex potential $u_{\omega}^{j}$ at $\mathbf{r}^{s}=\mathbf{r}-s \mathbf{n}(\mathbf{r})$ can be expressed as

$$
u_{\omega}^{j}\left(\mathbf{r}^{s}\right)=-\int_{\Omega} \nabla \Phi\left(\mathbf{r}^{s}, \mathbf{r}^{\prime}\right) \cdot \nabla u_{\omega}^{j}\left(\mathbf{r}^{\prime}\right) d \mathbf{r}^{\prime}
$$


where $\Phi\left(\mathbf{r}, \mathbf{r}^{\prime}\right)$ is the Neumann function of the Laplace operator in the domain $\Omega$. Denoting the background conductivity at the angular frequency $\omega$ by $\alpha_{\omega}^{b}=\left.\gamma_{\omega}\right|_{\Omega \backslash \bar{D}}$, we have

$$
u_{\omega}^{j}\left(\mathbf{r}^{s}\right)=-\frac{1}{\alpha_{\omega}^{b}} \int_{\Omega} \gamma_{\omega}\left(\mathbf{r}^{\prime}\right) \nabla \Phi\left(\mathbf{r}^{s}, \mathbf{r}^{\prime}\right) \cdot \nabla u_{\omega}^{j}\left(\mathbf{r}^{\prime}\right) d \mathbf{r}^{\prime}+\frac{1}{\alpha_{\omega}^{b}} \int_{D}\left[\gamma_{\omega}\left(\mathbf{r}^{\prime}\right)-\alpha_{\omega}^{b}\right] \nabla \Phi\left(\mathbf{r}^{s}, \mathbf{r}^{\prime}\right) \cdot \nabla u_{\omega}^{j}\left(\mathbf{r}^{\prime}\right) d \mathbf{r}^{\prime}
$$

Using $\gamma_{\omega_{1}} \frac{\partial u_{\omega_{1}}^{j}}{\partial \mathbf{n}} \approx \gamma_{\omega_{2}} \frac{\partial u_{\omega_{2}}^{j}}{\partial \mathbf{n}}$ on $\partial \Omega$ and taking $s \rightarrow 0^{+}$, we get

$$
\alpha_{\omega_{2}}^{b} u_{\omega_{2}}^{j}(\mathbf{r})-\alpha_{\omega_{1}}^{b} u_{\omega_{1}}^{j}(\mathbf{r}) \approx \int_{D} \nabla \Phi\left(\mathbf{r}, \mathbf{r}^{\prime}\right) \cdot\left[\tau_{2}\left(\mathbf{r}^{\prime}\right) \nabla u_{\omega_{2}}^{j}\left(\mathbf{r}^{\prime}\right)-\tau_{1}\left(\mathbf{r}^{\prime}\right) \nabla u_{\omega_{1}}^{j}\left(\mathbf{r}^{\prime}\right)\right] d \mathbf{r}^{\prime}, \quad \mathbf{r} \in \partial \Omega
$$

where $\tau_{l}=\gamma_{\omega_{l}}-\alpha_{\omega_{l}}^{b}, l=1,2$. This approximation provides a relationship between the anomaly $D$ and the weighted difference $\alpha_{\omega_{2}}^{b} \mathbb{U}_{\omega_{2}}-\alpha_{\omega_{1}}^{b} \mathbb{U}_{\omega_{1}}$ in such a way that

$$
\alpha_{\omega_{2}}^{b} U_{\omega_{2}}^{j, k}-\alpha_{\omega_{1}}^{b} U_{\omega_{1}}^{j, k} \approx \int_{D} \nabla \Phi\left(\mathbf{r}_{k}, \mathbf{r}^{\prime}\right) \cdot\left[\tau_{2}\left(\mathbf{r}^{\prime}\right) \nabla u_{\omega_{2}}^{j}\left(\mathbf{r}^{\prime}\right)-\tau_{1}\left(\mathbf{r}^{\prime}\right) \nabla u_{\omega_{1}}^{j}\left(\mathbf{r}^{\prime}\right)\right] d \mathbf{r}^{\prime}, \quad k, j=1,2, \ldots, L,
$$

where $\mathbf{r}_{k}$ is the center position of the electrodes $\mathcal{E}_{k}$. Replacing $\nabla u_{\omega}^{j}$ by a scalar multiple of $\nabla u_{0}^{j}$, we have

$$
U_{\omega_{2}}^{j, k}-\alpha U_{\omega_{1}}^{j, k} \approx \int_{D} \nabla \Phi\left(\mathbf{r}_{k}, \mathbf{r}^{\prime}\right) \cdot\left(\beta_{j}\left(\mathbf{r}^{\prime}\right) \nabla u_{0}^{j}\left(\mathbf{r}^{\prime}\right)\right) d \mathbf{r}^{\prime}, \quad k=1,2, \ldots, L
$$

where $\alpha=\frac{\alpha_{\omega_{1}}^{b}}{\alpha_{\omega_{2}}^{b}}$ and we use the rough approximation $\beta_{j} \nabla u_{0}^{j} \approx \frac{1}{\alpha_{\omega_{2}}^{b}}\left[\tau_{2} \nabla u_{\omega_{2}}^{j}-\tau_{1} \nabla u_{\omega_{1}}^{j}\right]$. In general, for any $\left(c_{1}, \ldots, c_{L}\right) \in \mathbb{R}^{L}$, we have

$$
\sum_{j=1}^{L} c_{j}\left[\mathbb{U}_{\omega_{2}}^{j, k}-\alpha \mathbb{U}_{\omega_{1}}^{j, k}\right] \approx \int_{D}\left[\nabla \Phi\left(\mathbf{r}_{k}, \mathbf{r}^{\prime}\right)-\nabla \Phi\left(\mathbf{r}_{k+1}, \mathbf{r}^{\prime}\right)\right] \cdot\left[\sum_{j=1}^{L} c_{j} \beta_{j}\left(\mathbf{r}^{\prime}\right) \nabla u_{0}^{j}\left(\mathbf{r}^{\prime}\right)\right] d \mathbf{r}^{\prime}, \quad k=1,2, \ldots, L
$$

We may refer to [19] for the above approximation.

The approximation (3) relates the row space of the matrix $\mathbb{U}_{\omega_{2}}-\alpha \mathbb{U}_{\omega_{1}}$ to the space spanned by the potential differences between adjacent electrodes of electric dipoles, $\nabla \Phi\left(\mathbf{r}_{k}, \mathbf{r}^{\prime}\right)-\nabla \Phi\left(\mathbf{r}_{k+1}, \mathbf{r}^{\prime}\right)$, situated in points $\mathbf{r}^{\prime}$ inside the anomaly $D$. This relation is exploited by the factorization method which was introduced by Kirsch [21] for inverse scattering problems and extended to EIT-problems by Brühl and Hanke in [6,7], see also [12,22] for further extensions. In the paper [14] we derive the following rigorous range criterion showing the feasibility of the factorization method to fdEIT:

Observation 1.1 ( [14]).

$$
z \in D \quad \text { if and only if }\left.\quad \nabla \Phi(\cdot, z)\right|_{\partial \Omega} \cdot \mathbf{d} \in \mathcal{R}\left(\left|\Re\left\{\alpha_{\omega_{2}}^{b} \Lambda_{\omega_{2}}-\alpha_{\omega_{1}}^{b} \Lambda_{\omega_{1}}\right\}\right|^{1 / 2}\right)
$$

where $\mathbf{d}$ is an unit vector, $\mathcal{R}(A)$ is the range of the operator $A$, and $\Lambda_{\omega}$ is the Neumann-to-Dirichlet operator at the angular frequency $\omega$.

Independently of the reconstruction method the utilization of differences of measured boundary voltages between chosen frequencies can alleviate undesirable effects of modelling errors. Figure 4(b) and (c) show frequency-difference images obtained with a single-step method based on a truncated singular value decomposition of the sensitivity matrix (see [31]). They clearly show that the fdEIT method is more robust against boundary geometry errors than the conventional static EIT method shown in figure 3. 


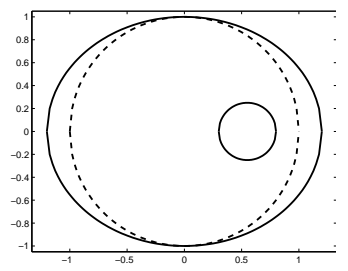

(a)

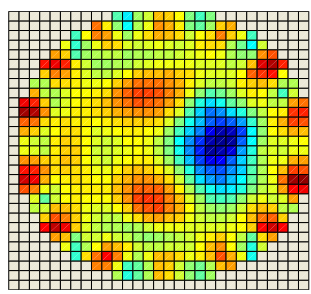

(b)

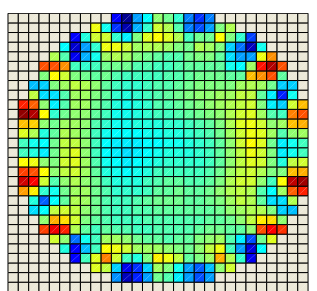

(c)

FiguRE 3. Effect of a boundary geometry error. (a) The ellipse with solid line was the true imaging domain and the circle in dashed line was the computational model domain. The small disk inside the domain was an anomaly. Complex conductivity values of the anomaly and the background were those of the banana and the saline, respectively. (b) and (c) are reconstructed static images (real-part images) at frequencies $\frac{\omega_{1}}{2 \pi}=100 \mathrm{~Hz}$ and $\frac{\omega_{2}}{2 \pi}=50 \mathrm{kHz}$, respectively. Each image was reconstructed using the boundary voltage data from the homogeneous computational model domain as the reference data. This figure is quoted from [31].

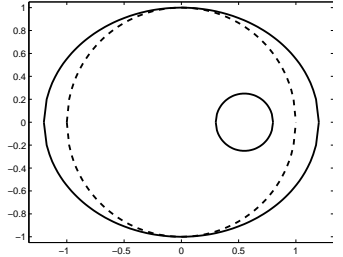

(a)

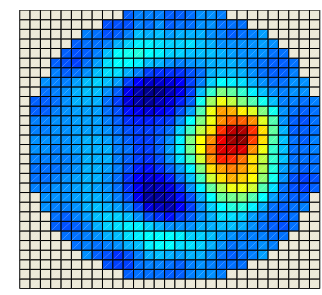

(b)

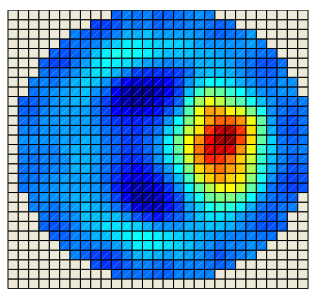

(c)

FIGURE 4. Robustness of the proposed fdEIT algorithm against a boundary geometry error. (a) The same true and computation domains explained in figure 3(a). (b) Real part of the reconstructed frequency-difference image $\Re\left\{\alpha_{b} \gamma_{\omega_{2}}-\gamma_{\omega_{1}}\right\}$ and (c) imaginary-part image $\Im\left\{\alpha_{b} \gamma_{\omega_{2}}-\gamma_{\omega_{1}}\right\}$. Two frequencies were $\frac{\omega_{1}}{2 \pi}=100 \mathrm{~Hz}$ and $\frac{\omega_{2}}{2 \pi}=50 \mathrm{kHz}$. The homogeneous computational model domain was used to compute the sensitivity matrix. This figure is quoted from [31].

Recently, using a 16-channel multi-frequency EIT (mfEIT) system KHU Mark1, we performed a fdEIT imaging experiment using a phantom with frequency-dependent complex conductivity distribution [18]. The results in [18] show that fdEIT is promising for imaging the complex conductivity contrast of an anomaly such as blood in hemorrhagic stroke and cancer tissue in breast. In [13], we carefully studied the practical implementation of the factorization method for the phantom experiment and justified a discrete version of observation 1.1 by relating it to the localized potentials in [11] that have high energy on some given subset of a domain and low energy on another.

Figure 5 shows the fdEIT phantom imaging experiment with a banana located in a background consisting of packed tiny pieces of carrots. Since the complex conductivity spectra of biological tissues show frequency dependency, we should use frequency-dependent materials as a background of phantom experiments. This means that we should not use a saline phantom including an biological anomaly which is too easy or worthless since the frequency-dependency would then only be present in the anomaly. 


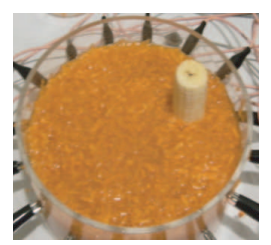

(a)

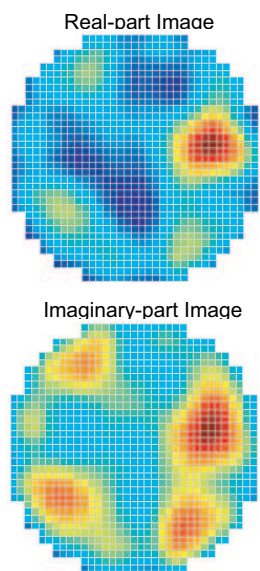

(b)

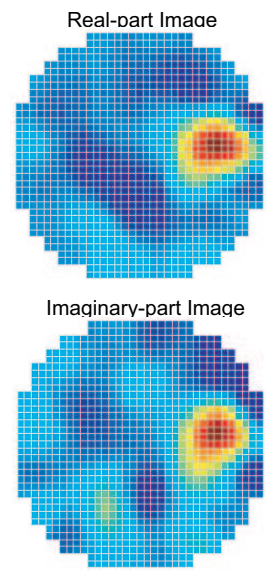

(c)

Figure 5. Phantom experiment of a homogeneous background with a frequency-dependent complex conductivity. (a) Picture of the phantom including banana in the background with packed tiny pieces of carrots. (b) and (c) are reconstructed fdEIT images using the simple difference $\mathbb{U}_{\omega_{2}}-\mathbb{U}_{\omega_{1}}$ and weighted voltage differences $\mathbb{U}_{\omega_{2}}-\alpha \mathbb{U}_{\omega_{1}}$, respectively. This figure is quoted from [18]

\section{FREQUenCy DiffEREnCE TAS}

In the paper [19], we apply the fdEIT method to a trans-admittance scanner (TAS), a device for detecting anomalies (such as breast cancer) whose conductivity is significantly different from surrounding tissues of normal conductivity $[2,3,17,30,32]$. On the surface of a region of a patient breast, we place a scanning probe with a planar array of electrodes kept at the ground potential and a patient holds a reference electrode with one hand through which a sinusoidal voltage $V_{0} \sin \omega t$ is applied. Let the human body occupy a three-dimensional domain $\Omega$ with a smooth boundary $\partial \Omega$. Let $\Gamma$ and $\gamma$ be portions of $\partial \Omega$, denoting the probe plane placed on the breast and the surface of the metallic reference electrode, respectively. As in the previous section, the resulting electric potential at a position $\mathbf{r}=(x, y, z)$ and time $t$ can be expressed as the real part of $u(\mathbf{r}) e^{i \omega t}$ where the corresponding complex potential $u_{\omega}$ at $\omega$ satisfies the following mixed boundary value problem:

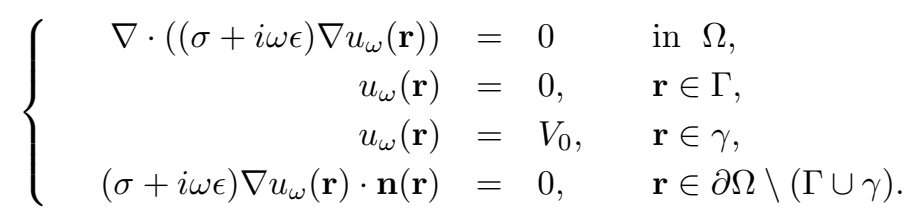

In multi-frequency TAS, we apply the voltage with two different frequencies $f_{1}=\omega_{1} / 2 \pi$ and $f_{2}=\omega_{2} / 2 \pi$ with $50 \mathrm{~Hz} \leq f_{1}<f_{2} \leq 500 \mathrm{kHz}$ and measure two sets of corresponding Neumann data $g_{1}=g_{\omega_{1}}$ and $g_{2}=g_{\omega_{2}}$ through $\Gamma$ at the same time. The inverse problem is to recover the anomaly such as breast cancer from the weighted difference between $g_{1}$ and $g_{2}$. In this section, we summarize some results in the paper [19] which provides a rigorous relation between the anomaly information and the weighted frequency difference. We denote the real and imaginary part of $u_{\omega_{j}}=u_{j}$ respectively by $\Re\left\{u_{j}\right\}=v_{j}$ and $\Im\left\{u_{j}\right\}=h_{j}$.

In order to detect a lesion $D$ underneath the scan probe $\Gamma$, we define a local region of interest under the probe plane $\Gamma$ as shown in Figure 6. Suppose that there is a cancerous lesion $D$ inside $\Omega_{L}$ and denote the complex 


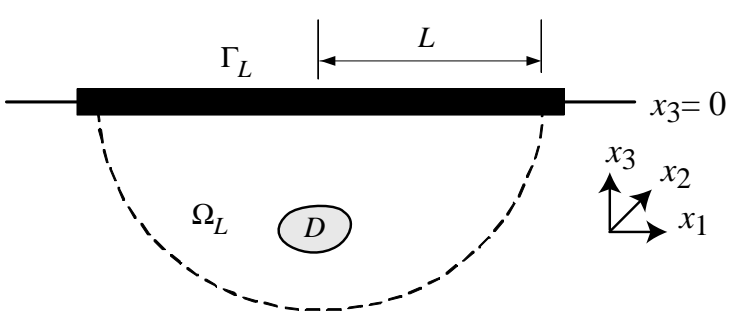

(a)

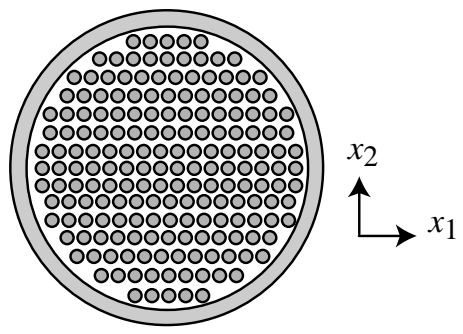

(b)

FiguRE 6. (a) Simplified model of the breast region with a cancerous lesion $D$ under the scan probe. (b) Schematic of the scan probe in the $\left(x_{1}, x_{2}\right)$-plane.

conductivity at the angular frequency $\omega_{j}$ by $\gamma_{j}=\sigma+i \omega_{j} \epsilon_{j}$. Assume $\gamma_{j}$ is a constant in $D$ and in $\Omega_{L} \backslash \bar{D}$;

$$
\sigma=\left\{\begin{array}{ll}
\sigma_{n} & \text { in } \Omega_{L} \backslash \bar{D} \\
\sigma_{c} & \text { in } D
\end{array} \quad \text { and } \quad \epsilon_{j}= \begin{cases}\epsilon_{j, n} & \text { in } \Omega_{L} \backslash \bar{D} \\
\epsilon_{j, c} & \text { in } D\end{cases}\right.
$$

For simplicity, we let $z$ be the axis normal to $\Gamma$ and let the center of $\Gamma$ be the origin. Hence, the probe region $\Gamma$ can be approximated as a two-dimensional region $\Gamma=\left\{(x, y, 0): \sqrt{x^{2}+y^{2}}<L\right\}$ where $L$ is the radius of the scan probe.

The following observation explains an explicit relation between $D$ and $\Im\left(g_{2}-\alpha g_{1}\right)$ where $\alpha=\frac{\sigma_{n}+i \omega_{2} \epsilon_{2, n}}{\sigma_{n}+i \omega_{1} \epsilon_{1, n}}$.

Observation 2.1 ( [19]). The imaginary part of the weighted difference $g_{2}-\alpha g_{1}$ satisfies the following formula:

$$
\begin{aligned}
\frac{1}{2 \sigma_{n}} \Im\left(g_{2}-\alpha g_{1}\right)(\mathbf{r}) & =\int_{D} \nabla_{\mathbf{r}^{\prime}} \frac{\partial \Phi\left(\mathbf{r}, \mathbf{r}^{\prime}\right)}{\partial z} \cdot \Theta\left(\mathbf{r}^{\prime}\right) d \mathbf{r}^{\prime} \\
& +\frac{\partial}{\partial z} \int_{\partial \Omega \backslash \Gamma} \frac{\partial \Phi\left(\mathbf{r}, \mathbf{r}^{\prime}\right)}{\partial z^{\prime}}\left[\int_{D} \nabla_{\tilde{\mathbf{r}}} \Psi\left(\mathbf{r}^{\prime}, \tilde{\mathbf{r}}\right) \cdot \Theta(\tilde{\mathbf{r}}) d \tilde{\mathbf{r}}\right] d s, \mathbf{r} \in \Gamma
\end{aligned}
$$

where

$$
\Theta(\mathbf{r})=\frac{\sigma_{n}-\sigma_{c}}{\sigma_{n}} \nabla\left(h_{2}-h_{1}\right)(\mathbf{r})+\frac{\omega_{2}\left(\epsilon_{2, n}-\epsilon_{2, c}\right)}{\sigma_{n}} \nabla\left(v_{2}-v_{1}\right)(\mathbf{r})-\Im\left(\beta \nabla u_{1}(\mathbf{r})\right),
$$

and

$$
\beta=\frac{i}{1+i \frac{\omega_{1} \epsilon_{1, n}}{\sigma_{n}}}\left[\frac{\omega_{2} \epsilon_{2, n}}{\sigma_{n}}\left(\frac{\epsilon_{2, c}}{\epsilon_{2, n}}-\frac{\sigma_{c}}{\sigma_{n}}\right)-\frac{\omega_{1} \epsilon_{1, n}}{\sigma_{n}}\left(\frac{\epsilon_{1, c}}{\epsilon_{1, n}}-\frac{\sigma_{c}}{\sigma_{n}}\right)-i \frac{\omega_{1} \omega_{2} \epsilon_{1, n} \epsilon_{2, n}}{\sigma_{n}^{2}}\left(\frac{\epsilon_{1, c}}{\epsilon_{1, n}}-\frac{\epsilon_{2, c}}{\epsilon_{2, n}}\right)\right] .
$$

In order to simplify the above representation formula, let us impose the following assumptions

$$
\begin{aligned}
& \bar{D} \subset \Omega_{L / 2}, \quad D=B_{\delta}(\xi), \quad \delta \leq \operatorname{dist}(D, \Gamma) \leq C_{1} \delta, \\
& \max \left\{\frac{\epsilon_{j, n}}{\epsilon_{j, c}}, \frac{\sigma_{n}}{\sigma_{c}}\right\} \leq \kappa_{1}, \quad \frac{\omega_{2} \epsilon_{2, n}}{\sigma_{n}} \leq \kappa_{2} \frac{\sigma_{n}}{\sigma_{c}}, \quad \frac{\sigma_{c}}{\sigma_{n}} \leq \kappa_{3},
\end{aligned}
$$

where $C_{1}$ is a positive constant, $B_{\delta}$ a ball with the radius $\delta$ and the center $\xi, \frac{\delta}{L} \leq \frac{1}{10}, \kappa_{1}$ and $\kappa_{2}$ are positive constants less than $\frac{1}{2}$ and $\kappa_{3}$ is a positive constant less than 10. Taking advantage of these assumption, we have the following observation.

Observation 2.2 ( [19]). The imaginary part of the weighted frequency difference $g_{2}-\alpha g_{1}$ can be expressed as

$$
\frac{1}{2 \sigma_{n}} \Im\left(g_{2}-\alpha g_{1}\right)(\mathbf{r})=\int_{D} \frac{\partial}{\partial z} \frac{\left(\mathbf{r}-\mathbf{r}^{\prime}\right) \cdot \tilde{\Theta}\left(\mathbf{r}^{\prime}\right)}{4 \pi\left|\mathbf{r}-\mathbf{r}^{\prime}\right|^{3}} d y+\operatorname{Error}(\mathbf{r}), \quad \mathbf{r} \in \Gamma_{L / 2},
$$



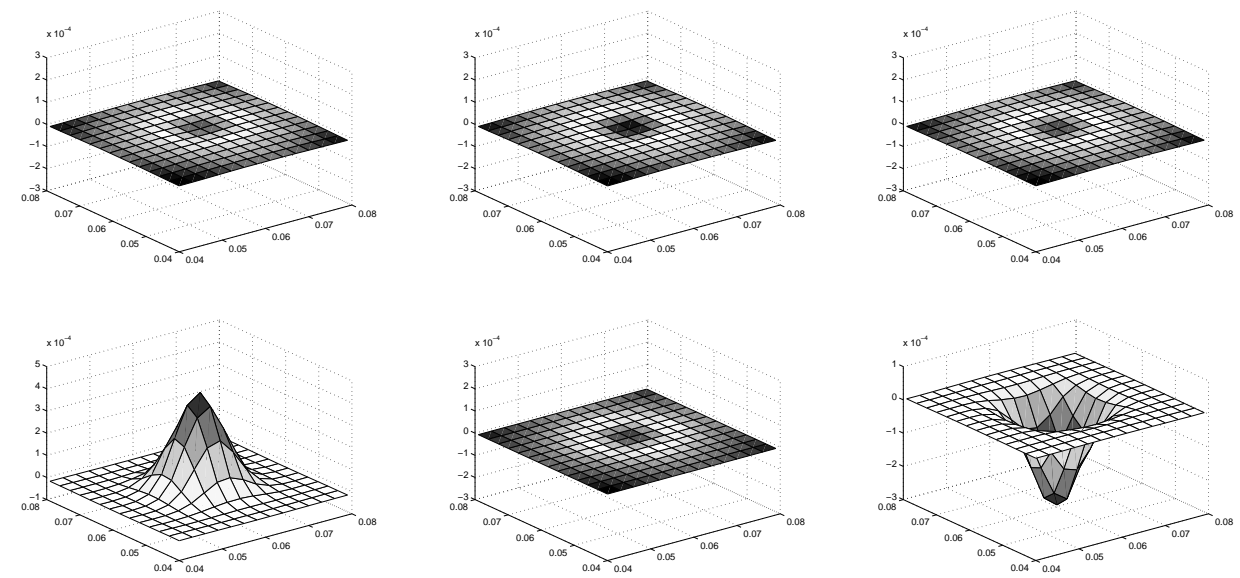

(a)

(b)

(c)

FIGURE 7. Frequency-difference trans-admittance map: real and imaginary parts of $g_{2}-\alpha g_{1}$

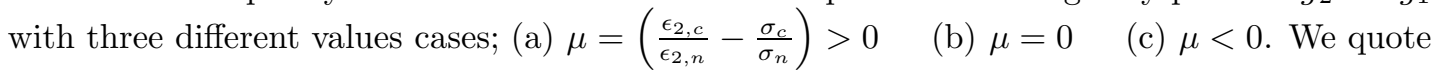
this figure from [19].

where

$$
\tilde{\Theta}=\frac{\sigma_{n}-\sigma_{c}}{\sigma_{n}} \nabla h_{2}-\frac{\omega_{2} \epsilon_{2, n}}{\sigma_{n}}\left(\frac{\epsilon_{2, c}}{\epsilon_{2, n}}-\frac{\sigma_{c}}{\sigma_{n}}\right) \nabla v_{1}
$$

and the error term Error(r) is estimated by

$$
\begin{aligned}
|\operatorname{Error}(\mathbf{r})| \leq & {\left[\frac{\omega_{2} \epsilon_{2, n}}{\sigma_{n}} \mathcal{P}_{1}\left(\left|\frac{\epsilon_{2, c}}{\epsilon_{2, n}}-\frac{\sigma_{c}}{\sigma_{n}}\right|\right) \frac{\delta^{3}}{L^{3}}\right.} \\
& \left.+\left(\frac{\omega_{1} \epsilon_{1, n}}{\sigma_{n}} \mathcal{P}_{1}\left(\left|\frac{\epsilon_{1, c}}{\epsilon_{1, n}}-\frac{\sigma_{c}}{\sigma_{n}}\right|\right)+\left(\frac{\omega_{2} \epsilon_{2, n}}{\sigma_{n}}\right)^{2} \mathcal{P}_{2}\left(\left|\frac{\epsilon_{2, c}}{\epsilon_{2, n}}-\frac{\sigma_{c}}{\sigma_{n}}\right|\right)\right) \frac{\delta^{3}}{|\mathbf{r}-\xi|^{3}}\right] .
\end{aligned}
$$

Here, $\mathcal{P}_{n}(\lambda)$ is a polynomial function of order $n$ such that $\mathcal{P}_{n}(0)=0$ and its coefficients depend only on $\kappa_{j}, j=1,2,3$.

In order to test the above observation, we consider a cubic model $\Omega:=[0,0.12] \times[0,0.12] \times[0,0.12] \mathrm{m}^{3}$ with the probe region $\Gamma:=\left\{(x, y, 0.12): \sqrt{x^{2}+y^{2}}<0.03\right\}$ and the reference electrode $\gamma:=\{(x, y, z) \in \Omega$ : $z=0\}$. Figure 7 shows the images of $g_{2}-\alpha g_{1}$ with three different values of $\omega_{2} \epsilon_{2, c}$ that are chosen so that the corresponding $\mu=\left(\frac{\epsilon_{2, c}}{\epsilon_{2, n}}-\frac{\sigma_{c}}{\sigma_{n}}\right)$ is positive, zero, or negative, respectively.

\section{Frequency differential Voltage Drop Method in NDE}

In [20], S. Kim et al. initiated a new remarkable nondestructive evaluation method, called fdEIS (frequency differential electrical impedance scanning), for evaluating the thickness of voids or cracks in conducting materials which is impossible in other conventional nondestructive methods. Finding the location of cracks and voids is relatively easy using any conventional NDE techniques such as ultrasonic testing, impact-echo, radiographic testing, electric potential drop method, and so on. However, all existing NDE techniques have inherent limitations in measuring the thickness of the cracks quantitatively.

To explain the fdEIS method in NDE, suppose that there is a crack inside a conducting material which occupies a rectangular parallelepiped region $\Omega$ with boundary $\partial \Omega$. Let $\Gamma$ and $\Upsilon$ be the portions of $\partial \Omega$ on which 


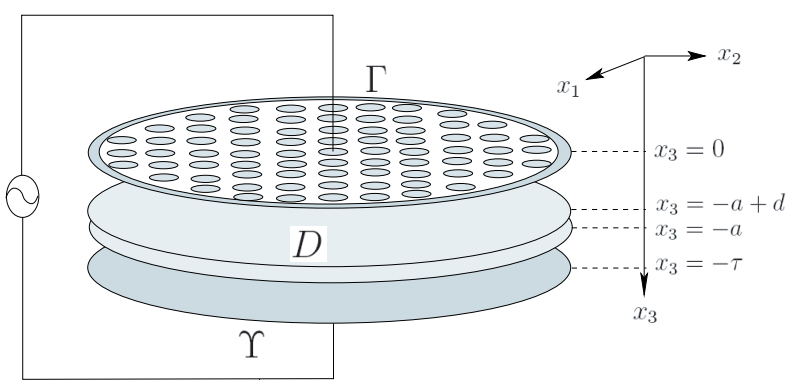

FIGURE 8. 1-D fdEIS Model

the scan probe and the reference electrode are placed, respectively, as in Figure 9. The basic mathematical model of fdEIS is the same as that of fdTAS in the previous section. We apply a sinusoidal voltage of $V_{0} \sin \omega t$ through $\Upsilon$ with the scan probe $\Gamma$ kept zero potential and measure the Neumann data $g_{\omega}$ on $\Gamma$ with respect to the angular frequency variable $\omega$.

In [20] S. Kim et al. found the following interesting relation between the thickness of the cracks and $\frac{d}{d \omega} g_{\omega}$, the derivative of the Neumann data with respect to angular frequency.

Observation 3.1 ( $[20])$. Assume $g_{\omega}$ is the measured Neumann data corresponding to the 1-D model shown in figure 8. Let $\tilde{\omega}=\omega \epsilon_{0}$. Then

$$
\left.\frac{d}{d \tilde{\omega}} \Im g_{\omega}(0)\right|_{\tilde{\omega}=0}=\left\{\begin{array}{cl}
V_{0} / d & \text { if } D \neq \emptyset . \\
0 & \text { if } D=\emptyset .
\end{array}\right.
$$

This fundamental principle in 1-D fdEIS explains how the frequency map $g_{\omega}$ is related with the thickness of the void $D$. This interrelation can be described more clearly by introducing the $\omega$-distance between $\Gamma$ and $\Upsilon$ defined by

$$
\operatorname{dist}_{\omega}(\Gamma, \Upsilon)=\frac{V_{0}}{\int_{\Gamma}\left|g_{\omega}\right| d s}
$$

Figure 9 shows how the $\omega$-distance between $\Gamma$ and $\Upsilon$ is related with the flow of the current density $(\sigma+i \omega \epsilon) \nabla u_{\omega}$.

\section{Conclusion}

Although there have been numerous research outcomes in EIT since early 1980s, the ill-posed nature of the inverse problem in EIT will continue to remain anyway and we should not expect EIT to compete with other medical imaging modalities in terms of spatial resolution. We have no hope in the static EIT without dealing with the forward modeling errors. To alleviate undesirable effects of modelling errors, we suggest fdEIT. The frequency dependent characteristics of biological tissue conductivity and permittivity are not available from any other imaging modality, fdEIT itself can be also quite promising for providing additional diagnostic information. Also, fdEIT method may find applications in various non-destructive evaluations such as crack evaluation, bubble detection, and others.

\section{REFERENCES}

1. G. Alessandrini, V. Isakov, and J. Powell, Local uniqueness in the inverse problem with one measurement, Trans. Amer. Math. Soc. 347 (1995), 3031-3041.

2. H. Ammari, O. Kwon, J. K. Seo, and E. J. Woo, T-scan electrical impedance imaging system for anomaly detection, SIAM J. Appl. Math. 65 (2004), 252-266.

3. M. Assenheimer, O. Laver-Moskovitz, D. Malonek, D. Manor, U. Nahliel, R. Nitzan, and A. Saad, The t-scan technology: Electrical impedance as a disgnostic tool for breast cancer detection, Physiol. Meas. 22 (2001), 1-8. 


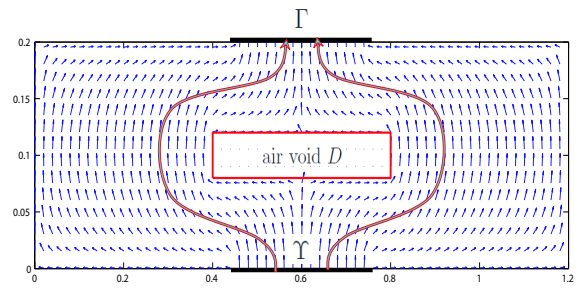

$(\mathrm{a}-1) \Re\left\{\left(\sigma+i \omega_{1} \epsilon\right) \nabla u_{\omega_{1}}\right\}$

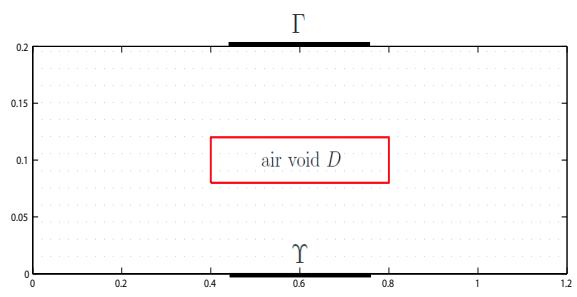

$(\mathrm{a}-2) \Im\left\{\left(\sigma+i \omega_{1} \epsilon\right) \nabla u_{\omega_{1}}\right\}$

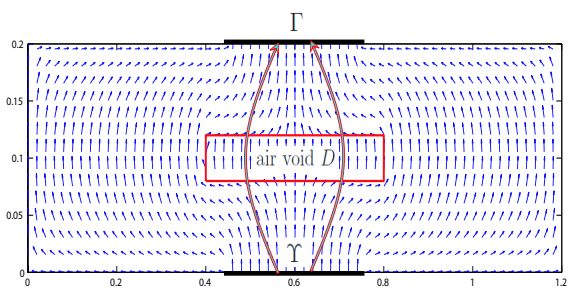

(b-1) $\left\{\left(\sigma+i \omega_{2} \epsilon\right) \nabla u_{\omega_{2}}\right\}$

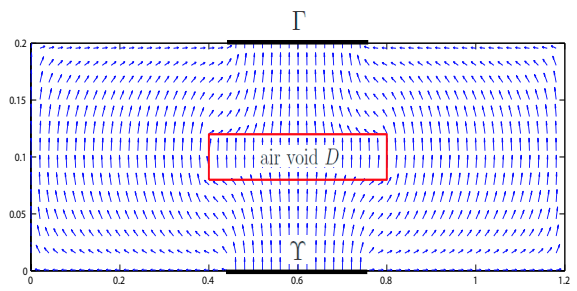

$(\mathrm{b}-2) \Im\left\{\left(\sigma+i \omega_{2} \epsilon\right) \nabla u_{\omega_{2}}\right\}$

FiguRE 9. The vector fields of real and imaginary parts of (a) $\left(\sigma+i \omega_{1} \epsilon\right) \nabla u_{\omega_{1}}$ with $\frac{\omega_{1}}{2 \pi}=10 \mathrm{~Hz}$ and (b) $\left(\sigma+i \omega_{2} \epsilon\right) \nabla u_{\omega_{2}}$ with $\frac{\omega_{2}}{2 \pi}=10^{5} \mathrm{~Hz}$. The curved arrows in (a-1) and (b-1) mean that the electrical current cannot pass through the void at the frequency $10 \mathrm{~Hz}$, while it can pass though the void at the higher frequency $10^{5} \mathrm{~Hz}$. This figure was quoted from [20].

4. D. C. Barber and B. H. Brown, Applied potential tomography, J. Phys. E. Sci. Instrum. 17 (1984), $723-733$.

5. K. Boone, D. Barber, and B. Brown, Imaging with electricity: report of the european concerted action on impedance tomography, J. Med. Eng. Tech 21 (1997), 201-232.

6. M. Brühl, Explicit characterization of inclusions in electrical impedance tomography, SIAM J. Math. Anal. 32 (2001), 13271341.

7. M. Brühl and M. Hanke, Numerical implementation of two non-iterative methods for locating inclusions by impedance tomography, Inverse Problems 16 (2000), 1029-1042.

8. M. Cheney, D. Isaacson, and J. C. Newell, Electrical impedance tomography, SIAM Review 41 (1999), 85-101.

9. M. Cheney, D. Isaacson, J. C. Newell, S. Simske, and J. Goble, Noser: An algorithm for solving the inverse conductivity problem, Int. J. Imaging Sys. and Tech. 2 (1990), 66-75.

10. A. Friedman and V. Isakov, On the uniqueness in the inverse conductivity problem with one measurement, Indiana Univ. Math. J. 38 (1989), 553-580.

11. B. Gebauer, Localized potentials in electrical impedance tomography, Inverse Probl. Imaging 2 (2008), 251-269.

12. M. Hanke and M. Brühl, Recent progress in electrical impedance tomography, Inverse Problems 19 (2003), S65-S90.

13. B. Harrach and J. K. Seo, Anomaly detection with frequency-difference electrical impedance tomography, preprint.

14. __ Detecting inclusions in electrical impedance tomography without reference measurements, SIAM J. Appl. Math. (submitted) (2009)

15. D. Holder, Electrical impedance tomography: Methods, history and applications, Bristol, UK, IOP Publishing (2005).

16. V. Isakov, On uniqueness of recovery of a discontinuous conductivity coefficient, Comm. Pure Appl. Math. 41 (1988), 856-877.

17. J. Jossinet and M. Schmitt, A review of parameters for the bioelectrical characterization of breast tissue, Ann. New York Academy of Sci. 873 (1999), 30-41.

18. S. C. Jun, J. Kuen, J. Lee, E. J. Woo, D. Holder, and J. K. Seo, Frequency-difference EIT (fdEIT) using weighted frequency difference and equivalent homogeneous complex conductivity: phantom imaging experiments, preprint.

19. S. Kim, J. Lee, J. K. Seo, E. J. Woo, and H. Zribi, Multi-frequency trans-admittance scanner: mathematical framework and feasibility, SIAM J. Appl. Math. (to appear).

20. S. Kim, J. K. Seo, and T. Ha, A nondestructive evaluation method of concrete voids: frequency differential electrical impedance scanning, SIAM J. Appl. Math. (submitted).

21. A. Kirsch, Characterization of the shape of a scattering obstacle using the spectral data of the far field operator, Inverse Problems 14 (1998), 1489-1512.

22. he factorization method for a class of inverse elliptic problems, Math. Nachr 278 (2005), $258-277$.

23. R. Kohn and M. Vogelius, Determining conductivity by boundary measurements, Comm. Pure Appl. Math. 37 (1984), $113-123$. 
24. P. Metherall, Three dimensional electrical impedance tomography of the human thorax, University of Sheffield, Dept. of Med. Phys and Clin. Eng., 1998.

25. P. Metherall, D. C. Barber, R. H. Smallwood, and B. H. Brow, Three-dimensional electrical impedance tomography, Nature 380 (1996), 509-512.

26. A. Nachman, Reconstructions from boundary measurements, Ann. Math. 128 (1988), 531-576.

27. Global uniqueness for a two-dimensional inverse boundary value problem, Ann. Math. 142 (1996), $71-96$.

28. T. I. Oh, W. Koo, K. H. Lee, S. M. Kim, J. Lee, S. W. Kim, J. K. Seo, and E. J. Woo, Validation of a multi-frequency electrical impedance tomography (mfEIT) system KHU Mark1: impedance spectroscopy and time difference imaging, Physiol. Meas. 29 (2008), 295-307.

29. G. J. Saulnier, R. S. Blue, J. C. Newell, D. Isaacson, and P. M. Edic, Electrical impedance tomography, IEEE Sig. Proc. Mag. 18 (2001), 31-43.

30. J. K. Seo, O. Kwon, H. Ammari, and E. J. Woo, Mathematical framework and anomaly estimation algorithm for breast cancer detection: electrical impedance technique using ts2000 configuration, IEEE Trans. Biomed. Eng. 51 (2004), 1298-1906.

31. J. K. Seo, J. Lee, H. Zribi, S. W. Kim, and E. J. Woo, Frequency-difference electrical impedance tomography (fdeit): Algorithm development and feasibility study, Physiol. Meas. 29 (2008), 929-944.

32. J. E. Silva, J. P. Marques, and J. Jossinet, Classification of breast tissue by electrical impedance spectroscopy, Med. Biol. Eng. Comput. 38 (2000), 26-30.

33. E. Somersalo, M. Cheney, and D. Isaacson, Existence and uniqueness for electrode models for electric current computed tomography, SIAM J. Appl. Math. 22 (1992), 1023-40.

34. J. Sylvester and G. Uhlmann, A uniqueness theorem for an inverse boundary value problem in electrical prospection, Comm. Pure Appl. Math. 39 (1986), 91-112.

35. _ A global uniqueness theorem for an inverse boundary value problem, Ann. Math. 125 (1987), $153-169$.

36. G. Uhlmann, Developments in inverse problems since calderdon's foundational paper, Harmonic analysis and partial differential equations (Chicago, IL, 1996), Univ. Chicago Press (1999), 295-345.

37. J. G. Webster, Electrical impedance tomography, Adam Hilger, Bristol, UK, 1990. 